\title{
Antibiotic resistance: challenges and successes in respiratory infection
}

\author{
Sanjay Sethi*,1 \& Jenny Bryan²

\section{European Respiratory Society Congress, Amsterdam, 26-30 September 2015, and CHEST 2015, Montréal, Canada, 24-28 October 2015}

With approximately 50,000 deaths in the USA and EU attributed to antibacterial resistance each year, together with several million days of hospital care $[1]$, the need to address resistance mechanisms and find new targets for novel antibiotics has never been greater. At the annual congresses of the European Respiratory Society and the American College of Chest Physicians, presenters reported advances in understanding of the mechanisms of antibiotic resistance and how these may be overcome. The latest clinical trial data on antibiotic treatment for hospital- and community-acquired pneumonia, including the potential for novel nebulized forms of therapy, were also discussed.

First draft submitted: 7 January 2016; Accepted for publication: 7 January 2016;

Published online: 15 April 2016

\section{Global trends in antibiotic resistance}

In the age of mass migration of refugees from countries with high antibiotic resistance rates, Tobias Welte (Hannover Medical School, Hannover, Germany) demonstrated why clinicians need to be aware of global variations in antibiotic resistance, and to base their choice of antibiotic on national guidelines that take account of changing local patterns of resistance.

Speaking at the European Respiratory Society (ERS) congress, he used data from the European Antimicrobial Resistance Surveillance Network (EARS-Net) to show that, while rates of penicillinresistant Streptococcus pneumoniae are low and stable across much of Europe, rising levels of macrolide resistance, especially in Southern and Eastern Europe (with levels up to 50\%), are cause for concern. Even more important, he said, is the increase in macrolide-resistant mycoplasma pneumonia, where the choice of alternative antibiotics is more limited than for $S$. pneumoniae. He reported recent data show macrolide-resistant mycoplasma pneumonia rates of $90 \%$ in Japan and China, and increasing levels in Canada, the USA and Europe. Welte described two recent, very severe cases of mycoplasma pneumonia in his own clinic, in refugees. Although both patients survived, he attributed the severity of infection to a combination of increased pathogenicity of the mycoplasma and the poor health of exhausted refugees. He warned that such cases will become more common, and that tuberculosis (TB) should be considered as a likely coinfection with community acquired pneumonia (CAP) in refugees from Asian countries with a high prevalence of TB. For methicillin-resistant Staphylococcus aureus (MRSA), the news is much better, with resistance having plateaued at approximately $20 \%$ in most European countries, including a big reduction in countries such as the UK and France where

\section{KEYWORDS}

- antibiotics

- antimicrobial resistance

- respiratory infections 
MRSA was previously a major problem. With this in mind, Welte urged delegates generally to consider a staphylococcal $\beta$-lactam antibiotic for $S$. aureus - not one of the newer drugs targeting resistant strains.

The picture is very different for Gramnegative (Gm-ve) infection. The rise in extended-spectrum $\beta$-lactam (ESBL) resistance started about 5 years ago and has led to a big increase in carbapenem usage, resulting in increased carbapenemase resistance. In Europe, highest rates are in Italy and Greece, with countries in the Middle East, Asia and South America also badly affected. In contrast to MRSA with its three resistance mechanisms, a new mechanism of carbapenemase resistance is published almost every week, said Welte, making it difficult to develop new antibiotics.

Completing his review, Welte pointed out that, despite the high mortality associated with Pseudomonas pneumonia, antibacterial resistance is not as high as for some other Gm-ve organisms. Of the older agents, ceftazidime remains effective in $80-85 \%$ of patients but ciprofloxacin should be avoided because of resistance.

\section{Antibiotic resistance: mechanisms \& drivers}

Infectious disease researchers will need to 'think outside the box' if they are to overcome the challenge of multidrug-resistant bacteria, according to Georgina Cox (McMaster University, Hamilton, Canada).

She reminded delegates at the ERS congress of the four key mechanisms of antibiotic resistance and the antibiotics most commonly affected [2]:

- Efflux mechanisms, e.g.: fluoroquinolones, aminoglycosides, tetracyclines, $\beta$-lactams, macrolides

- Immunity and bypass, e.g.: tetracyclines, trimethoprim, sulphonamides, vancomycin

- Target modification, e.g.: fluoroquinolones, rifamycins, vancomycin, penicillins, macrolides, aminoglycosides

- Inactivating enzymes, e.g.: $\beta$-lactams, aminoglycosides, macrolides, rifamycins

In her discussion of the antibiotic resistome, Cox explained that, in addition to intrinsic resistance, bacteria may have silent or protoresistant mechanisms that allow them to acquire resistance. Bacteria that start with silent resistance have genes that can cause resistance in the presence of an appropriate promoter while protoresistant organisms have determinants capable of evolving into resistant enzymes under pressure of antibiotic prescribing. Cox presented results of recent protoresistance research showing the potential of DNA polymerase- $\beta$ enzymes, which normally play a 'housekeeping' role in DNA repair and excision, to evolve into resistance enzymes when exposed to antibiotics [3].

Cox suggested that screening for adjuvants to resensitise resistant organisms to commonly used antibiotics holds considerable promise. Such adjuvants have no antibacterial effects but they potentiate the activity of subinhibitory concentrations of antibiotics. Cox discussed recently published research showing that the fungal natural product, aspergillomarasmine $A$ (AMA), inhibits the activity of the metallo- $\beta$ lactamase (MBL), NDM-1, which is commonly responsible for resistance to carbapenems [4]. In a series of experiments, adjuvant AMA treatment was shown to restore the activity of meropenem against Enterobacteriaceae, Acinetobacter spp. and Pseudomonas spp. with VIM or NDM-type alleles. Survival of mice infected with Klebsiella pneumoniae expressing NDM-1 was greater in animals treated with AMA and meropenem than in those treated with AMA or meropenem alone.

Cox also described using the anthracycline antibiotic 301A, isolated from a fermentation broth of Streptomyces, to sensitize Gm-ve bacteria (Escherichia coli and Acinetobacter baumannii) to rifampicin and linezolid - antibiotics to which they are usually intrinsically resistant [5]. Further analysis showed that the adjuvant antibiotic exerted this effect by inhibiting the efflux mechanisms of bacterial cells.

\section{Newer agents for hospital-acquired pneumonia \& CAP}

Optimizing doses and combinations of newer antibiotics is essential for prolonging their activity against multiresistant organisms, agreed speakers at both the ERS and CHEST meetings.

Rosario Menéndez Villanueva (Hospital Universitario La Fe, Valencia, Spain) highlighted research showing the importance of using the higher $100 \mathrm{mg}$ dose of tigecycline in order to achieve sufficient concentrations for clinical cure in hospital-acquired pneumonia (HAP) [6], and suggested that more studies are needed to confirm the optimal dose. She explained that the vancomycin derivative, telavancin, which is very active against multiresistant strains of Gram 
positive $(\mathrm{Gm}+\mathrm{ve})$ bacteria, with rapid concentration and good distribution in the lung, has shown noninferiority to vancomycin in HAP, though increased creatinine is more common [7].

Menéndez Villanueva reported that dalbavancin has aroused considerable interest because of its unusual dosing, thanks to its prolonged half-life. Patients are treated with $1000 \mathrm{mg}$ of dalbavancin on day 1 and $500 \mathrm{mg} 1$ week later, and there is no need for dose modification in renal failure, but this agent is currently licensed only in skin and soft tissue infection. Against multiresistant Gm-ve pathogens, the cephalosporin/ $\beta$ lactam combinations, ceftolozane/tazobactam and ceftazidime/avibactam have shown in vitro activity against Enterobacteriaceae and Pseudomonas aeruginosa, and ceftazidime/avibactam has demonstrated consistent activity against Klebsiella pneumoniae carbapenemase (KPC)producing organisms [8]. Menéndez Villanueva added that both combinations are currently in ongoing Phase III trials in ventilator-associated pneumonia (VAP) and nosocomial pneumonia.

Turning to novel treatments for CAP, Menéndez Villanueva drew delegates' attention to the two new cephalosporins, ceftaroline and ceftobiprole, the nonfluorinated quinolone, nemonoxacin and the fourth-generation macrolide, solithromycin. She explained that ceftaroline is active against $\mathrm{Gm}+\mathrm{ve}$ bacteria, including MRSA, and some Gm-ve organisms, but not Pseudomonas. Ceftaroline has been shown to be noninferior to ceftriaxone in CAP, with numerically higher clinical cure rates and a similar safety profile [9]. Menéndez Villanueva said that ceftobiprole is also effective in MRSA and has the advantage of being active against $P$. aeruginosa. However, it is not active against ESBL-producing Enterobacteriaceae. In patients with CAP severe enough to require hospitalisation, ceftobiprole was shown to be noninferior to ceftriaxone \pm linezolid, clinically and microbiologically [10]. Menéndez Villanueva added that nemonoxacin is active against CAP pathogens, including MRSA, but appears to be more effective in communityacquired than hospital-acquired MRSA. In a comparative study in CAP patients, oral nemonoxacin 750 and $500 \mathrm{mg}$ administered for 7 days resulted in high clinical and bacteriological success rates in CAP patients and was shown to be as effective as levofloxacin $500 \mathrm{mg}$ [11].

Results of the Solitaire-Oral study, presented at CHEST by Dr Carlos Barrera (A and L Clinical Research, CA, USA) [12], and at the ERS meeting, showed that treatment with the fourth generation macrolide, solithromycin (800 mg on day 1 and solithromycin $400 \mathrm{mg}$ on days 2-5) was noninferior to moxifloxacin $400 \mathrm{mg}$ for 7 days in patients with CAP. Numerically higher early response rates and success at short-term follow-up were seen with solithromycin than moxifloxacin in elderly patients and those with chronic obstructive pulmonary disease (COPD) or asthma. Safety outcomes for the two drugs were comparable.

Dr Barrera explained that the structure of solithromycin is based on that of clarithromycin, but with additional chemical moieties designed to interact with bacterial ribosomes at three different sites, each of which would need to be overcome or bypassed for bacterial resistance to develop. Solithromycin is being developed in both oral and iv. formulations for the treatment of CAP, and an exploratory trial evaluating its effects in reducing airway inflammation in patients with COPD is ongoing.

\section{What role for aerosolized antibiotics?}

Aerosolized antibiotics hold considerable promise in the treatment of bacterial infection in critically ill patients, but concerns about their potential for driving resistance, and uncertainty over optimum methods of administration continue to limit their use.

At CHEST, Marcos Restrepo (University of Texas Health Science Center at San Antonio, TX, USA) presented data suggesting that, far from increasing resistance problems, aerosolized antibiotics can actually help to eradicate multidrugresistant organisms [13]. Results of a double-blind placebo-controlled study in 47 critically ill, intubated patients showed that aerosolized antibiotics were associated with eradication of 26/27 organisms present at randomization. This compared with $2 / 23$ organisms in the placebo group ( $p<$ $0.0001)$. Resistant organisms were eradicated in 14/16 patients treated with aerosolized antibiotics compared with $1 / 11$ in the placebo group $(p<0.001)$. New resistant organisms were seen in $2 / 16$ patients on aerosolized antibiotics versus $6 / 11$ in the placebo group ( $p=0.03$ ).

Restrepo pointed out that results contrasted with the findings of a previous meta-analysis suggesting that aerosolized antibiotic prophylaxis may increase resistant bacteria [14]. But he proposed that the combination of aerosolized and systemic treatment used in the more recent study may have contributed to the successful drive against resistant organisms [15]. 
Michael Niederman (New York Presbyterian Hospital and Weill Cornell Medical Center, NY, USA) explained that optimizing distal deposition of aerosolized antibiotics in the lungs in patients with HAP or ventilator associated pneumonia is the key to minimizing intensive care unit contamination and risk of development of resistant pathogens. While accepting that it can be difficult to achieve peripheral delivery in patients with pneumonia, Niederman said small particle formulations of antibiotics $(<5 \mathrm{~nm})$, delivered by vibrating mesh nebulizers co-ordinated with the ventilatory cycle, appear to achieve deepest delivery. He added that the depth of delivery is likely to improve with continued treatment as the peripheral airways become clear and penetration is improved. He concluded that, as the technology continues to evolve, clinicians will need to work closely with respiratory therapy departments to ensure that each patient gets the antibiotic formulation and delivery device best suited to their needs.

\section{Financial \& competing interests disclosure}

$S$ Sethi has received consulting fees from Cempra and Aradigm, and speaker honoraria from Bayer.J Bryan's attendance at the ERS and CHEST conferences and her contribution to the writing of the report were supported by Cempra. The authors have no other relevant affliations or financial involvement with any organization or entity with a financial interest in or financial conflict with the subject matter or materials discussed in the manuscript apart from those disclosed.

No writing assistance was utilized in the production of this manuscript.

\section{References}

1 WHO. Antimicrobial Resistance. Global Report on Surveillance 2014. http://www.who.int/drugresistance/ documents/surveillancereport/en/

2 Wright GD. Q\&A: antibiotic resistance: where does it come from and what can we do about it? BMC Biol. 8, 123 (2010).

3 Cox G, Stogios PJ, Savchenko A, Wright GD Structural and molecular basis for resistance to aminoglycoside antibiotics by the adenylyltransferase ANT(2")-Ia. MBio 6(1) (2015).

4 King AM, Reid-Yu SA, Wang W et al. Aspergillomarasmine A overcomes metallo- $\beta$ lactamase antibiotic resistance. Nature 26510(7506), 503-506 (2014).

5 Cox G, Koteva K, Wright GD. An unusual class of anthracyclines potentiate Grampositive antibiotics in intrinsically resistant Gram-negative bacteria. J. Antimicrob. Chemother. 69(7), 1844-1855 (2014).

-6 Ramirez J, Dartois N, Gandjini H et al. Randomized Phase 2 trial to evaluate the clinical efficacy of two high-dosage tigecycline regimens versus imipenemcilastatin for treatment of hospital-acquired pneumonia. Antimicrob. Agents Chemother. 57(4), 1756-1762 (2013).

$\$$ Rubinstein E, Lalani T, Corey GR et al. ATTAIN Study Group. Telavancin versus vancomycin for hospital-acquired pneumonia due to gram-positive pathogens. Clin. Infect. Dis. 52(1), 31-40 (2011).

\section{-8 Liscio JL, Mahoney MV, Hirsch EB.} Ceftolozane/tazobactam and ceftazidime/ avibactam: two novel $\beta$-lactam/ $\beta$-lactamase inhibitor combination agents for the treatment of resistant Gram-negative bacterial infections. Int. J. Antimicrob. Agents 46(3), 266-271 (2015).

-9 File TM Jr, Low DE, Eckburg PB et al. Integrated analysis of FOCUS 1 and FOCUS 2: randomized, doubled-blinded, multicenter Phase 3 trials of the efficacy and safety of ceftaroline fosamil versus ceftriaxone in patients with community-acquired pneumonia. Clin. Infect. Dis. 51(12), 1395-1405 (2010).

10 Nicholson SC, Welte T, File TM Jr et al. A randomised, double-blind trial comparing ceftobiprole medocaril with ceftriaxone with or without linezolid for the treatment of patients with community-acquired pneumonia requiring hospitalisation. Int. J. Antimicrob. Agents 39(3), 240-246 (2012).

-11 van Rensburg DJ, Perng RP, Mitha IH et al. Efficacy and safety of nemonoxacin versus levofloxacin for community-acquired pneumonia. Antimicrob. Agents Chemother. 54(10), 4098-4106 (2010).

12 Oldach D, Barrera C, Rowe B et al. Results from a Phase 3 trial in moderate to moderately severe community acquired bacterial pneumonia (CABP) treated as outpatients with a new oral macrolide, solithromycin. Chest 148(4), Abstract 78A (2015).

13 Palmer LB, Smaldone GC. Reduction of bacterial resistance with inhaled antibiotics in the intensive care unit. Am. J. Respir. Crit. Care Med. 189(10), 1225-1233 (2014).

- 14 Falagas ME, Siempos II, Bliziotis IA, Michalopoulos A. Administration of antibiotics via the respiratory tract for the prevention of ICU-acquired pneumonia: a meta-analysis of comparative trials. Crit. Care 10(4), R123 (2006).

$\checkmark 15$ Restrepo MI, Keyt H, Reyes LF. Aerosolized antibiotics. Respir. Care. 60(6), 761-762 (2015). 